(SC35, SRp40, SRp55, SRp20 and hnRNPA1), and the ratios between exon 4 inclusion/exclusion were evaluated by RT-PCR. Second, we examined the effects of different cytokines on $\triangle 4 \mathrm{BAFF}$ induction.

Results RAMOS cells presented exon 4 skipping (ratio inclusion/ exclusion: 6.8) after minigene transfection. Following co-transfection of the minigene with coding plasmids for splicing proteins, only the overexpression of SC35 showed effect in the splicing of exon 4, promoting exon 4 inclusion (ratio: $>30$ ). Incubation of different cell lines with several cytokines showed that IFN- $\gamma$ was able to induce $\Delta 4 \mathrm{BAFF}$-transcript. Thus, after IFN- $\gamma$ stimulation in the minigene model, the ratio inclusion/exclusion markedly decreased (1.5). IFN- $\gamma$ modifies the balance between SC35 and another member of hnRNPs family (hnRNP C1/C2) favouring the alternative splicing of exon 4.

Conclusions These results demonstrated that IFN- $\gamma$ induces $\triangle 4 \mathrm{BAFF}$, modifying the function of SC35 protein and increasing the hnRNPC1/C2. Our study provides an expanded conceptual view of BAFF gene regulation, and contributes to a better understanding of the mechanisms involved in BAFF up-regulation in autoimmunity.

\section{A5.11 DETECTION OF ACPA PRODUCING B-CELLS BY A CITRULLINE PEPTIDE PANEL}

doi:10.1136/annrheumdis-2013-203219.11

'Eszter Szarka, ${ }^{1}$ Krisztina Huber, ${ }^{1} J u d i t$ Pozsgay, ${ }^{2}$ Fruzsina Babos, ${ }^{3}$ Tamás Gáti, ${ }^{2}$ Anna Magyar, ${ }^{2}$ Ferenc Hudecz, ${ }^{3}$ Bernadette Rojkovich, ${ }^{3}$ György Nagy, ${ }^{1,4}$ Gabriella Sármay. ${ }^{1}$ Department of Immunology, Eötvös Loránd University, Budapest; ${ }^{2}$ HAS-ELTE Research Group of Peptide Chemistry, Budapest; ${ }^{3}$ Buda Hospital of Hospitaller Brothers of St. John, Budapest; ${ }^{4}$ Immunology Research Group of the Hungarian Academy of Sciences at ELTE, Budapest, Hungary

Background and Objectives Anti-citrullinated protein/peptide antibodies (ACPAs) are the most sensitive and specific serological markers of RA. To identify the optimal epitopes that detect different subgroups of RA patients with high sensitivity and specificity, we have investigated citrulline and arginine containing peptides derived from filaggrin, collagen or vimentin. We have identified a citrulline-containing peptide panel that was recognised by RA sera with high specificity. Our aim was to compare this peptide panel with the conventionally used serological assays and to detect peptide-specific ACPA producing B-cells in in vitro cultures.

Materials and Methods Previously selected citrulline- and arginine-containing filaggrin, vimentin and collagen peptide epitopes were investigated. We compared the recognition of these peptides by RA and control sera using indirect ELISA. B-cells were purified from peripheral blood by negative selection, IgG production was stimulated by B-cell activators (R848 and recombinant human IL-2) provided with the human ELISPOT kit. Antibody producing cells were enumerated after 4 days culture by using peptide-specific ELISPOT assay.

Results Sera samples from 247 RA and 148 age-matched $(57 \pm 14$ years) healthy controls were collected. The citrulline peptide panel detected approximately $80 \%$ of RA patients, including $20 \%$ of seronegative/CCP negative patients as well. Individual peptides detected different subgroups of RA patients. The more peptides recognised by a particular RA serum sample, the more severe the disease of the patient was. In vitro cultured B-cells from selected RA patients synthesised multiple citrulline-containing peptide-specific antibodies after polyclonal stimulation, while B-cells from healthy blood donors did not.

Conclusions The citrulline peptide panel can detect $20 \%$ of ACPA negative RA patients thus may have a prognostic value. Furthermore, the panel is suitable to detect citrulline peptide-specific antibody producing cells, thus enables us to study ACPA producing B-cells of RA patients.

\section{A5.12 DISAPPEARANCE AND REAPPEARANCE OF IGG, IGA AND IGM AUTOANTIBODY ISOTYPES AND IMMUNE COMPLEXES IN RITUXIMAB-TREATED SLE PATIENTS}

doi:10.1136/annrheumdis-2013-203219.12

'Barbro Persson, 'Fionnuala McAree, ${ }^{2}$ Malin Müller, ${ }^{2}$ Tina Trollmo, ${ }^{2}$ loannis Parodis, ${ }^{3}$ Thorunn Jonsdottir, ${ }^{2}$ Vivianne Malmström, ${ }^{2}$ Iva Gunnarsson, 'Johan Rönnelid. 'Department of Immunology, Genetics and Pathology, Uppsala University, Uppsala, Sweden, ' Unit of Rheumatology, Department of Medicine, Karolinska Institutet, Stockholm, Sweden; ${ }^{3}$ Department of Rheumatology, Landspitali University Hospital, Reykjavik, Iceland

Background and Objectives We have earlier investigated the content of specific IgG autoantibodies in SLE immune complexes (IC; Åhlin et al, Lupus 2012; 21:586). For that purpose we have developed a line blot technique for the quantification also of nonclassical (IgA and IgM) SLE autoantibody isotypes. In SLE patients, IgG autoantibody levels drop after institution of Rituximab therapy. The objective was to investigate parallel changes IgG, IgA and IgM autoantibody isotypes in parallel to IC levels.

Materials and Methods Nine SLE patients initially treated with two infusions of Rituximab were followed with repeated samplings at baseline and after 1, 3, 6 and 12 months. Thawed samples were investigated simultaneously concerning rheumatoid factor (RF) isotypes and C1q-binding IC with enzyme immunoassays. All samples from patients with ANA-associated autoantibodies (6/9) were investigated concerning IgG/A/M autoantibodies with line blot quantitated with densitometry and concerning IgG autoantibodies with ALBIA/Luminex technique. Significant changes were defined either as $\geq 33 \%$ drop or as $\geq 50 \%$ increase, compared to the lowest levels experienced during the follow-up period.

Results ALBIA measurements showed significant initial drop in anti-dsDNA in 4/6 patients but also significant drop in levels of anti-histone, anti-SSA/Ro60, anti-Sm and anti-Sm/RNP in individual patients. Late increases in IC and antibodies against dsDNA, SSA/Ro52, SSA/Ro60, SSB, Sm, Sm/RNP ribosomal P protein and histones were associated with clinical relapse. Late increase in IgA/ IgM anti-DNA, anti-histones and anti-nucleosomes was also found in one patient with persistent kidney disease treated with mycophenolate mofetil at 10 months. Non-classical autoantibody isotypes showed late increases that often were not paralleled by the corresponding IgG autoantibodies. Two patients showed late increase in RF isotypes in parallel to clinical relapse. Different autoantibodies/isotypes showed different kinetics of appearance/disappearance. All ANA autoantibody positive patients initially had increased IC levels, which dropped significantly after therapy in $4 / 6$ patients. The autoantibody negative patients never had increased IC levels and showed no significant changes in RF.

Conclusions Measurement of non-classical isotypes of RF and ANA-associated autoantibodies might yield clinically useful information when monitoring SLE patients treated with B cell depleting therapy.

\section{A5.13 EFFECT OF RITUXIMAB ON B CELL SUBPOPULATIONS EXPRESSING THE 9G4 IDIOTYPE IN PATIENTS WITH RHEUMATOID ARTHRITIS}

doi:10.1136/annrheumdis-2013-203219.13

${ }^{1,2}$ Rita A Moura, ${ }^{2,3}$ Inmaculada de la Torre, ${ }^{2}$ Maria J Leandro, ${ }^{2}$ Geraldine Cambridge. ${ }^{1}$ Rheumatology Research Unit, Instituto de Medicina Molecular, Lisbon, Portugal, ${ }^{2}$ Centre for Rheumatology Research, Division of Medicine, University College London, London, UK; ${ }^{3}$ Rheumatology Department, Gregorio Marañón Hospital, Madrid, Spain

Background and Objectives Antibodies encoded by the $V_{H} 4-34$ gene are inherently autoreactive, binding to red blood cell 